\title{
Emission lines from tidally disrupted white dwarfs and other evolved stars
}

\author{
D. Clausen ${ }^{1, a}$, M. Eracleous ${ }^{1}$, S. Sigurdsson ${ }^{1}$, and J.A. Irwin ${ }^{2}$ \\ ${ }^{1}$ Department of Astronomy \& Astrophysics, The Pennsylvania State University, 525 Davey Lab, University Park, \\ PA 16802, USA \\ ${ }^{2}$ Department of Physics and Astronomy, University of Alabama, Box 870324, Tuscaloosa, AL 35487, USA
}

\begin{abstract}
When a black hole tidally disrupts a star, accretion of the debris will produce a luminous flare and reveal the presence of a dormant black hole. The accretion flare can also photoionize a portion of the post-disruption debris. We present models of the emission line spectrum produced in the debris released when a white dwarf or a horizontal branch star is tidally disrupted by an intermediate-mass black hole, and discuss the possibility of using the emission lines to identify such events and constrain the properties of the black hole. We also compare the white dwarf disruption models with observations of white dwarf tidal disruption candidates in globular clusters associated with NGC 4472 and NGC 1399. The bright [O III] lines observed in each system are consistent with these models, but there are some drawbacks to interpreting these sources as tidally disrupted white dwarfs. On the other hand, models of the emission line spectrum produced when a horizontal branch star is disrupted by a $\sim 100 \mathrm{M}_{\odot}$ black hole are in good agreement with the source in the NGC 1399 globular cluster. Finally, we describe light curves for the emission lines produced in the debris of a tidally disrupted helium core. The modeled light curves are consistent with the recent observations of Gezari et al. (2012).
\end{abstract}

\section{INTRODUCTION}

Several luminous UV/X-ray flares observed in the cores of normal galaxies have been identified as candidate tidal disruption events (TDEs) [e.g., 1, 2]. The spectra and light curves of these flares are consistent with theoretical expectations for the emission produced when a black hole $(\mathrm{BH})$ accretes a portion of a tidally disrupted star $[3,4]$. Theory also predicts that in most cases a significant fraction of the post-disruption debris will not fall back to the BH. In particular, if the disrupted star was initially on a parabolic orbit, then at least half of the debris will remain unbound from the $\mathrm{BH}$ after tidal disruption [5]. This debris tail will be photoionized by the accretion flare, resulting in an emission line spectrum that differentiates TDE flares from other transient events such, as supernovae or AGN outbursts. The emission lines produced in the debris of a solar type star that has been disrupted by a supermassive BH have been studied using both numerical [6] and analytic [7] models. These investigations found that the emission line spectra, which are dominated by hydrogen lines, could be used to characterize the disruption scenario.

$\mathrm{BHs}$ in the cores of galaxies or globular clusters (GCs) can also tidally disrupt evolved stars that pass too closely $[8,9]$. Of particular interest are white dwarf (WD) tidal disruptions. For deeply penetrating disruptions, tidal compression of the WD can ignite nuclear reactions resulting in an explosive energy release [10]. Furthermore, for BHs more massive than about $3 \times 10^{5} \mathrm{M}_{\odot}$, the tidal disruption radius for a WD is within the BH's event horizon. This means that there will only be a luminous accretion flare if the disruptor is an intermediate mass black hole (IMBH). In addition to serving as signposts for IMBHs, WD tidal disruptions are potential multimessenger sources because they produce gravitational

a e-mail: dclausen@astro.psu.edu radiation $[11,12]$. To aid in the identification of WD TDEs, we modeled the distinctive emission line spectra radiated by the stream of unbound debris created when a WD is disrupted by an IMBH. Motivated by discrepancies between our models and candidate WD TDEs, and by reports of a stripped helium core TDE, we have also modeled the emission lines produced in the debris of tidally disrupted horizontal branch (HB) stars and the exposed core of a red giant.

\section{THE MODEL}

Details of the method used to compute the emission line luminosities and profiles are discussed in [13]. The techniques are an adaptation of those developed by Strubbe \& Quataert [7]. We considered stars that were initially on parabolic orbits with pericenter distance $R_{P}$ less than the tidal disruption radius $R_{T}=R_{\star}\left(M_{\mathrm{BH}} / M_{\star}\right)^{1 / 3}$. Here, $R_{\star}$ and $M_{\star}$ are the radius and mass of the star, respectively, and $M_{\mathrm{BH}}$ is the mass of the $\mathrm{BH}$. We assumed that after tidal disruption, half of the shredded star would fall back to pericenter at a rate $\dot{M} \propto t^{-5 / 3}$, and form an accretion disk around the $\mathrm{BH}$. The accretion rate in the disk was set to the mass fallback rate. When the accretion rate was super-Eddington, which was always the case shortly after disruption, we assumed that the excess was blown away in a radiatively driven wind. However, we did not include the effects of this wind in our models. Using $\dot{M}$ and the radial extent of the disk, we computed the disk's temperature profile and constructed the multicolor blackbody emitted by the disk.

In the model, the remaining material is assumed to flow away from the $\mathrm{BH}$ with a range of velocities given by

$$
v(\phi)=\left(\frac{2 G M_{\mathrm{BH}}}{R_{\mathrm{P}}}\right)^{1 / 2} \cot \left(\frac{\phi}{2}\right),
$$


and densities given by $n(\phi, t) \propto[v(\phi) t]^{-3}$. Here, $\phi$ is the azimuthal angle in the orbital plane and is in the range $\pi-\left(12 R_{\star} / R_{\mathrm{P}}\right)^{1 / 2}<\phi<\pi$. We split the debris tail into six azimuthal sections and computed photoionization models for each section with Cloudy [14]. We used the multicolor blackbody emitted by the disk as the input ionizing spectral energy distribution (SED). In some models, we experimented with adding an X-ray power law to the SED and found that this addition did not affect the lines of interest. Combining the emission line fluxes calculated in the photoionization models with the velocity field of the unbound debris tail allowed us to synthesize emission line profiles.

\section{EMISSION LINES FROM TIDALLY DISRUPTED WHITE DWARFS}

In [13], we modeled the emission lines formed in the debris of tidally disrupted WDs. In all of the models we used a WD comprising, by mass, $67 \%$ oxygen, $32 \%$ carbon, and $1 \%$ helium and other elements with their relative abundances scaled from the solar values. The WD had $M_{\star}=0.55 \mathrm{M}_{\odot}, R_{\star}=8.6 \times 10^{8} \mathrm{~cm}$.

The brightest emission lines in this scenario are the C IV $\lambda \lambda 1548,1550$ and [O III] $\lambda \lambda 4959,5007$ doublets. The C IV doublet is the brightest feature at early times with peak luminosity $L_{\mathrm{C} \text { IV }} \sim 10^{38} \mathrm{erg} \mathrm{s}^{-1}$. As the debris tail expands and becomes less dense, the C IV luminosity declines. The evolution of the [O III] luminosity is slightly more complex. While the density in most of the debris tail is above the critical density for [O III] $\lambda \lambda 4959,5007$ $\left(n_{c}=7 \times 10^{5} \mathrm{~cm}^{-3}\right)$, the doublet becomes brighter as the debris tail expands. The [O III] doublet reaches its peak luminosity of $L_{[\mathrm{O}} \mathrm{II]}=$ few $\times 10^{37} \mathrm{erg} \mathrm{s}^{-1}$ when the most distant section of the debris tail reaches the transition's critical density.

The models predict that the [O III] lines should be quite broad, with full width at half maximum (FWHM) that can be as large as $2500 \mathrm{~km} \mathrm{~s}^{-1}$. Furthermore, the synthesized emission line profiles are highly asymmetric, with extended blue wings.

We found that properties of the IMBH-WD system are encoded in the emission lines. When $R_{P}=R_{T}$, the [O III] luminosity peaks about 2 years after tidal disruption for IMBHs in the mass range $100 \mathrm{M}_{\odot}<M_{\mathrm{BH}}<10^{4} \mathrm{M}_{\odot}$. For stronger encounters, $R_{P}<R_{T}$, the [O III] feature will reach its peak luminosity within months of tidal disruption. The peak [O III] $\lambda 5007$ luminosity depends weakly on $\mathrm{BH}$ mass, with lower mass BHs leading to higher [O III] luminosities. In addition to being weak, this dependance is degenerate with $\mathrm{BH}$ spin. Thus, we conclude that it would be difficult to determine the mass and/or spin of the disrupting $\mathrm{BH}$ from the emission lines. However, bright, broad, asymmetric C IV and [O III] lines can be used to identify WD tidal disruptions when they are observed in concert with an X-ray/UV flare in the core of a galaxy or GC.

\section{COMPARISON TO OBSERVATIONS}

There are two extragalactic GCs that host ultraluminous $\mathrm{X}$-ray sources (ULXs) and exhibit bright optical emission lines. One of these ULXs, in a GC associated with NGC 4472, undergoes large luminosity variations that suggest that the accretor is a $\mathrm{BH}$ [15]. Optical spectroscopy of the GC hosting this ULX revealed an [O III] $\lambda 5007$ emission line with $L=1.4 \times 10^{37} \mathrm{erg} \mathrm{s}^{-1}$ and FWHM $=$ $1500 \mathrm{~km} \mathrm{~s}^{-1}$, but did not detect any hydrogen emission lines [16]. The other ULX, in a GC associated with NGC 1399, has a softer spectrum than typical ULXs, which suggests that this source is also an accreting $\mathrm{BH}$ [17]. An optical spectrum of the GC hosting this ULX showed [O III] $\lambda 5007$ and [N II] $\lambda 6583$ emission lines, each with $\mathrm{L} \sim$ few $\times 10^{36} \mathrm{erg} \mathrm{s}^{-1}$. Interestingly, there are no hydrogen lines in this spectrum either. However, the emission lines seen in the NGC 1399 GC are much narrower than those observed in the NGC 4472 GC, with FWHM $=140 \mathrm{~km} \mathrm{~s}^{-1}$. Based on the high X-ray luminosities, bright [O III] lines, and the lack of hydrogen features in these sources, Irwin et al. [17] suggested that they could be WD TDEs.

How do these sources compare with the models? Both the luminosity and FWHM of the [O III] lines observed in the NGC 4472 source are consistent with the models presented in [13]. In the models, this high [O III] luminosity is reached two years after disruption and only persists for a matter of months before declining. The X-ray source in the NGC 4472 cluster, however, had been on for at least 14 years at the time of the optical observations [18]. This timescale mismatch disfavors interpreting this source as a WD TDE. The luminosity of the [O III] feature seen in the NGC 1399 cluster is also consistent with the models. However, its relatively narrow width, and the presence of an equally bright $\mathrm{N}[\mathrm{II}]$ line are difficult to reconcile with models of WD disruption. In all of the WD tidal disruption models that we computed, the [N II] line was at least two orders of magnitude fainter than the [O III] line. Given the discrepancies between the observations and the models, we conclude that it is difficult to interpret either source as a WD TDE.

\section{EMISSION LINES FROM TIDALLY DISRUPTED HORIZONTAL BRANCH STARS}

Even though observations of the NGC 1399 ULX are not completely compatible with the WD tidal disruption models, the X-ray luminosity and emission lines do resemble the signatures of a TDE. Motivated by the similarities, we considered the disruption of other types of evolved stars. Several properties of HB stars make them favorable candidates. During the star's main sequence lifetime, CNO cycle burning depletes hydrogen and enriches the core with nitrogen at the expense of carbon and oxygen. Then, mass loss during the red giant phase removes a significant portion of the star's envelope, amplifying the core's contribution to the overall composition of the star. The result is a nitrogen rich and hydrogen poor abundance pattern, which seems to be implied by the observed emission lines. Furthermore, although they are of comparable mass, the radius of a HB star is $\sim 300$ times larger than that of a WD. Accordingly, for a given $M_{\mathrm{BH}}, R_{T}$ is much larger for $\mathrm{HB}$ stars than WDs, and the range of velocities in the debris tail is much smaller (see Eqn. 1). The decreased velocity range could 


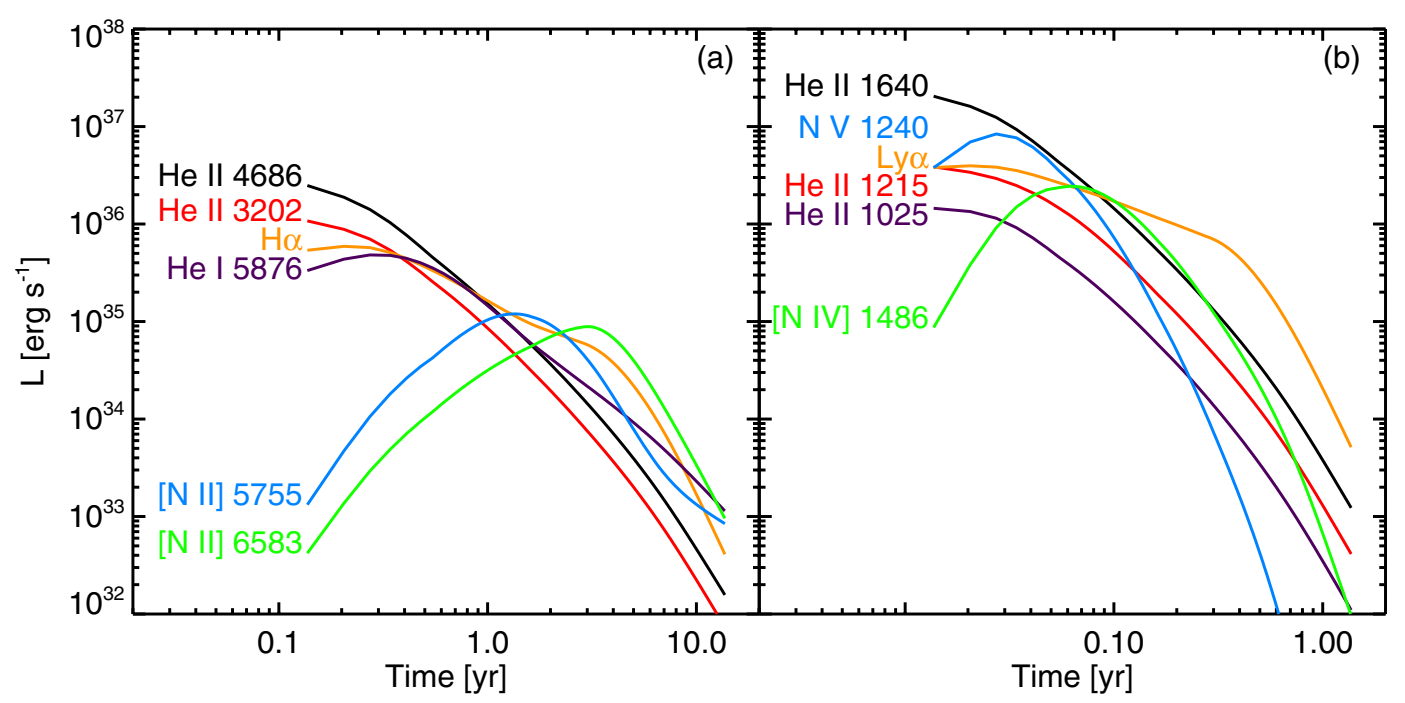

Figure 1. Model emission line light curves for the photoionized debris of a tidally disrupted, stripped helium core. Here we have used $M_{\star}=0.4 \mathrm{M}_{\odot}, R_{\star}=0.13 \mathrm{R}_{\odot}$, and $M_{\mathrm{BH}}=10^{6} \mathrm{M}_{\odot}$. Optical/near-UV emission lines are shown in panel (a). Early on, He II $\lambda 4686$ and He II $\lambda 3202$ are the brightest lines, which is consistent with the observations reported in [21]. Panel (b) shows the UV emission line light curves.

account for the narrower emission lines observed in the NGC 1399 GC.

We briefly describe our HB star tidal disruption models here and refer the reader to [19] for a complete description. We determined the mass, radius, and composition of an HB star using the stellar evolution code MESA STAR [20]. To bracket the range of likely outcomes, we created models of both a red clump HB star, typical of metal rich GCs, and an extreme HB star, typical of a metal poor GC. The model red clump HB star had $M_{\star}=0.67 \mathrm{M}_{\odot}, R_{\star}=6.2 \mathrm{R}_{\odot}$, and comprised $15 \%$ hydrogen, $84 \%$ helium, $0.1 \%$ carbon, $0.5 \%$ nitrogen, and $0.2 \%$ oxygen, by mass. We considered BHs in the mass range $30-10^{4} \mathrm{M}_{\odot}$ and tidal disruption parameters $\beta=R_{T} / R_{p}$ in the range $1-10$.

In all of the models that we computed, the photoionized debris emitted [N II] $\lambda 6583$ and [O III] $\lambda 5007$ lines with $L \sim 10^{36} \mathrm{erg} \mathrm{s}^{-1}$; values consistent with the observed luminosities of these features. However, requiring that the models match the observed emission line ratios, [N II]/ $\mathrm{H} \alpha>7$, [O III] $/ \mathrm{H} \beta>5$, and $[\mathrm{N} \mathrm{II}] /[\mathrm{O} \mathrm{III}] \sim 1$, for an extended period of time allowed us to constrain $\beta, M_{\mathrm{BH}}$, and the nature of the HB star. Within the tidal disruption scenario, we found that the data are best explained as the mild $(\beta=1)$ disruption of a red clump $\mathrm{HB}$ star by a $\mathrm{BH}$ with $M_{\mathrm{BH}} \leq 200 \mathrm{M}_{\odot}$.

The fact that the models favor a red clump HB star is appealing because the cluster hosting the ULX is quite red, and likely exhibits the red horizontal branch morphology typical of a metal rich cluster. However, some aspects of this interpretation of the data are problematic. First, helium burning in the HB star creates carbon and oxygen and destroys nitrogen. There is only a period of roughly 50 Myr during which an HB star can be disrupted and leave a debris tail with a nitrogen abundance large enough to produce the observed emission lines. Furthermore, N-body models suggest that main sequence TDEs should occur far more frequently than HB star TDEs in GCs [9]. If this source is a HB TDE, then why haven't several main sequence TDEs also been observed in GCs?
A possible solution to these problems is for the HB star to be bound to the $\mathrm{BH}$ in a binary. This situation presents two scenarios for tidal disruption, either a scattering induced merger or a triple decay. In each, the system begins as a $\mathrm{BH}$ with a main sequence companion. When the companion evolves up the red giant branch, it fills its Roche lobe and begins transferring mass to the $\mathrm{BH}$. The mass transfer results in orbital expansion, creating a wide binary. In the scattering induced merger case, this expansion greatly increases the likelihood that the binary will interact with another star in the GC. Such an encounter can alter the binary and bring the evolved star too close to its $\mathrm{BH}$, resulting in a tidal disruption. In the triple decay scenario, the binary is the inner component of a hierarchical triple. The orbital expansion drives the system to instability, triggering the tidal disruption. In both situations, the chain of events that leads to the tidal disruption of the star is precipitated by that star's evolution to the red giant phase. Hence, these scenarios favor the disruption of post main sequence stars.

One way to test the HB TDE interpretation is with UV spectroscopy. The models predict several UV emission lines including N V $\lambda \lambda 1239,1243$, O VI $\lambda \lambda 1032,1038$, N IV] $\lambda 1486$, and C IV $\lambda \lambda 1548,1550$. If these lines are present, they will be additional evidence in support of the HB TDE hypothesis and can be used to further constrain properties of the BH. Their absence, on the other hand, would be difficult to explain in the context of the TDE model.

\section{EMISSION LINES FROM A TIDALLY DISRUPTED, STRIPPED HE CORE}

The optical transient PS1-10jh has been interpreted as the tidal disruption of the stripped core of an evolved star [21]. The UV-optical light curve for the source is well sampled and displays the distinctive $t^{-5 / 3}$ decline expected for TDE flares. Interestingly, an optical spectrum taken 22 
rest-frame days before the peak in the light curve contains two emission lines, He II $\lambda 4686$ and He II $\lambda 3202$.

We have explored the interpretation of the data presented in [21] with a set of emission line models. In the models presented here, we used $M_{\mathrm{BH}}=10^{6} \mathrm{M}_{\odot}$. We constructed a stripped He core model using the "stellar engineering" capabilities in MESA STAR. We considered the extreme case in which almost the entire hydrogen-rich envelope was removed. To do this, a $1 \mathrm{M}_{\odot}$ star with $Z=$ 0.001 was evolved to the red giant branch. Before the star reached the tip of the red giant branch and ignited $\mathrm{He}$, we stopped its evolution and manually removed the outer layers of the star until the envelope mass was $10^{-3} \mathrm{M}_{\odot}$. The resulting star had $M_{\star}=0.41 \mathrm{M}_{\odot}, R_{\star}=0.13 \mathrm{R}_{\odot}$, and consisted of $99 \%$ helium.

The predicted emission line light curves are shown in Figure 1. Initially, the brightest lines are He II $\lambda 4686$ and He II 13202. These are the two emission lines seen in the optical spectrum of PS1-10jh taken 22 rest frame days before the peak in the optical light curve. In the models, $\mathrm{H} \alpha$ and He I $\lambda 5876$ begin to outshine the He II $\lambda 3202$ within a few months of tidal disruption. A year after tidal disruption, the brightest emission lines are [N II] $\lambda 5755$ and [N II] 26583 . There is no evidence for this evolution in the spectra presented in [21], but this is not surprising. A year after tidal disruption, the brightest emission lines are an order of magnitude fainter than the He II lines seen shortly after disruption, and even these He II lines were barley detected. These more detailed models confirm the conclusion of Gezari et al. [21], who interpreted PS1-10jh as the tidal disruption of a bare He core.

As was the case with the HB TDE discussed above, the photoionized debris of the disrupted He core should emit several, bright UV lines. The modeled light curves for the UV emission lines are shown in panel (b) of Figure 2. The UV lines may be easier to detect than the optical lines because they have higher luminosities. However, continuum emission from the accretion flare is also stronger in the UV, and this may overwhelm the line emission. Nonetheless, UV spectroscopy of PS1-10jh could better constrain the nature of this candidate TDE.

Along with additional data, further modeling of the source's emission line spectrum is required. We have only investigated the tidal disruption of a completely bare He core. In reality, the core would likely retain a larger portion of the hydrogen-rich envelope. More importantly, we have only modeled the emission line luminosities, not the emission line profiles. The observed profiles have FWHM $=9000 \mathrm{~km} \mathrm{~s}^{-1}$, which is substantially smaller than the range of velocities in the debris tail. Furthermore, the observed emission lines are not red or blue shifted with respect to the host galaxy. These discrepancies may be due to our line of sight to the source, but synthetic line profiles are required to resolve this issue.

\section{References}

[1] S. Komossa, N. Bade, A\&A 343, 775 (1999)

[2] S. Gezari, S. Basa, D.C. Martin, G. Bazin, K. Forster, B. Milliard, J.P. Halpern, P.G. Friedman, P. Morrissey, S.G. Neff et al., ApJ 676, 944 (2008)

[3] M.J. Rees, Nature 333, 523 (1988)

[4] A. Ulmer, ApJ 514, 180 (1999)

[5] J.H. Lacy, C.H. Townes, D.J. Hollenbach, ApJ 262, 120 (1982)

[6] T. Bogdanović, M. Eracleous, S. Mahadevan, S. Sigurdsson, P. Laguna, ApJ 610, 707 (2004)

[7] L.E. Strubbe, E. Quataert, MNRAS 400, 2070 (2009)

[8] S. Sigurdsson, M.J. Rees, MNRAS 284, 318 (1997)

[9] H. Baumgardt, J. Makino, T. Ebisuzaki, ApJ 613, 1143 (2004)

[10] S. Rosswog, E. Ramirez-Ruiz, W.R. Hix, ApJ 695, 404 (2009)

[11] A. Sesana, A. Vecchio, M. Eracleous, S. Sigurdsson, MNRAS 391, 718 (2008)

[12] R. Haas, R.V. Shcherbakov, T. Bode, P. Laguna, ApJ 749, 117 (2012)

[13] D. Clausen, M. Eracleous, ApJ 726, 34 (2011)

[14] G.J. Ferland, K.T. Korista, D.A. Verner, J.W. Ferguson, J.B. Kingdon, E.M. Verner, PASP 110, 761 (1998)

[15] T.J. Maccarone, A. Kundu, S.E. Zepf, K.L. Rhode, Nature 445, 183 (2007)

[16] S.E. Zepf, D. Stern, T.J. Maccarone, A. Kundu, M. Kamionkowski, K.L. Rhode, J.J. Salzer, R. Ciardullo, C. Gronwall, ApJL 683, L139 (2008)

[17] J.A. Irwin, T.G. Brink, J.N. Bregman, T.P. Roberts, ApJL 712, L1 (2010)

[18] T.J. Maccarone, A. Kundu, S.E. Zepf, K.L. Rhode, MNRAS 409, L84 (2010)

[19] D. Clausen, S. Sigurdsson, M. Eracleous, J.A. Irwin, MNRAS 424, 1268 (2012)

[20] B. Paxton, L. Bildsten, A. Dotter, F. Herwig, P. Lesaffre, F. Timmes, ApJS 192, 3 (2011)

[21] S. Gezari, R. Chornock, A. Rest, M.E. Huber, K. Forster, E. Berger, P.J. Challis, J.D. Neill, D.C. Martin, T. Heckman et al., Nature 485, 217 (2012) 